\title{
Airborne SAR - ATI - GMTI Influence Factor Analysis
}

\author{
Weidong Zhang \\ Air Force Aviation University \\ Chang Chun, JiLin, China \\ zwd1970@163.com
}

\author{
Nuan Song \\ Air Force Aviation University \\ Chang Chun, JiLin , China \\ bluebirdsong@163.com
}

\author{
Jianfeng Zhang \\ Air Force Aviation University \\ Chang Chun , JiLin , China \\ zjfengce@126.com
}

\begin{abstract}
This paper focuses on the airborne SAR double channel data using ATI method into the Moving Target Indication process various factors affecting the discussion. Based on the actual flight appeared in the process of nonideal motion state and the engineering application of the factors such as detailed analysis, only understand the influence factors to determine the effective detection method, simplified data processing process, and improve the data processing efficiency.
\end{abstract}

Keywords- Synthetic Aperture Radar, Ground Moving Target Indication, Along Track Interferometry, Influence

\section{INTRODUCTION}

Synthetic Aperture Radar (SAR) along the track interferometry(ATI ${ }^{[1]}$ ground moving target Indication (GMTI) is one of the most commonly used method of SARGMTI, whether in military reconnaissance ${ }^{[2]}$ or civil transportation monitoring ${ }^{[3]}$, all plays a very important role.

According to the SAR-ATI research in theoretical aspects, there are many reports in the literature ${ }^{[4-5]}$ at home and abroad, but also put forward a lot of solution, but most of them are built on the basis of simulation data, this paper focuses on the airborne SAR double channel data using ATI method into the Moving Target Indication process various factors affecting the discussion. in view of the practical application in engineering problems are analyzed in detail, and gives the corresponding solution, and achieved good results, and to the ground moving target had good heavy positioning and speed. Finally, according to the paper aims to ATI-GMTI quick solutions, simplifies some airborne SAR data processing steps, is easy to realize airborne SAR processing equipment real-time GMTI.

\section{SAR-ATI PERFORMANCE THEORY ANALYSIS}

Method using the ATI is the interference phase of two channel image data to detect moving target, it has no high requirements to two channel energy equalization, as long as the moving target radial velocity does not fall in the blind speed area, through the phase threshold method can carry on the detection. For the airborne system which is single emission and gathered much, it makes use of installation on the same platform, along the path direction arrangement, interval $L$ of 2 times baseline length $B$ of two pairs of aerial to Measurement and draw the ground scene. If the ground target is static, then the two antennas is for the same image, and moving target will be in the corresponding pixels produce phase shift. After the two echo signal antenna obtained respectively imaging processing, form the interference phase diagram, phase difference $\Delta \Phi$ and doppler frequency shift $\omega_{\mathrm{d}}$ and time interval $\Delta t$ are relevant, $\Delta \Phi=\omega_{\mathrm{d}} \bullet \Delta t$, namely the relationship is proportional to radial velocity, use the information of $\Delta \Phi$, it can be detecting the action target through the corresponding processing means. Figure 1 gives SAR-ATI space geometrical relationship.

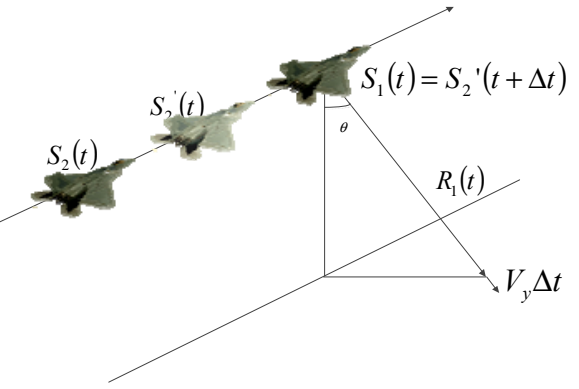

Figure 1. SAR-ATI space geometrical relationship diagram

Hypothesis antenna $S_{1}$ transmitting signals, antenna $S_{1}$ and $S_{2}$ receiving echo, according to the principle of equivalent phase centers, antenna $S_{1}$ and $S_{2}$ can respectively send and receive the electromagnetic wave which can be equivalent to $S_{1}$ and $S_{2}$ connecting line in the middle there is a equivalent antenna $S_{2}$ is both send and receive, then to the ground the same motion target two antenna receives the echo phase is:

$$
\begin{aligned}
& \phi_{1}=-\frac{2 \pi}{\lambda} \cdot 2 R_{1}(t) \\
& \phi_{2}=-\frac{2 \pi}{\lambda} \cdot 2 \cdot R_{2}{ }^{\prime}(t+\Delta t)
\end{aligned}
$$

After the intervention of the phase difference is:

$$
\begin{aligned}
& \Phi_{21}=\phi_{2}-\phi_{1}=-\frac{4 \pi}{\lambda}\left[R_{2}{ }^{\prime}(t+\Delta t)-R_{1}(t)\right]=-\frac{4 \pi}{\lambda} \cdot V_{y} \Delta t \\
& \Phi_{12}=-\Phi_{21}
\end{aligned}
$$

Among them, $V_{\mathrm{y}}$ is the moving target is the radial (along the radar line of sight direction) speed, $\lambda$ is wavelength. So:

$$
V_{y}=\frac{1}{\Delta t}\left(\frac{\lambda}{4 \pi} \Phi_{12}\right)
$$

In order to avoid phase happen aliasing fuzzy, $\Phi_{21}$ should be in between[- $\pi, \pi]$, at the same time, $\Phi_{21}$ also must be greater than a certain minimum phase detection 
threshold $\Phi$ base (correspond to the minimum radial detectable speed), therefore can get system detection blind speed regional expression:

$$
V_{\text {blind }} \in\left[\frac{K \lambda v}{B_{\rightarrow}}-\frac{\Phi_{\text {base }} \lambda v}{2 \pi B_{\rightarrow}}, \frac{K \lambda v}{B_{\rightarrow}}+\frac{\Phi_{\text {base }} \lambda v}{2 \pi B_{\rightarrow}}\right], \quad K \in Z
$$

In the blind speed area, can't detect moving target, between $\mathrm{k}=0$ and 1 from two blind speed is non-fuzzy detectable speed range.

\section{ThE ACTUAL FLIGHT STATE ON SAR-ATI NOW WE KNOW ATI'S INFLUENCE}

ATI moving target indication is the basis of the ground observation area of imaging, and the working principle of SAR for radar platform in imaging term, keep constant, linear motion, and the actual flight condition is load machine by airflow disturbances, the airplane movement condition and ideal state of movement there is a big error, some of these error on imaging quality produced great influence, some for interferometric phase produced great influence. Below, we will analysis by detailed about these motion error.

Here is a presupposition, the airplane is a mechanical structure good rigid body, installation in the same plane platform on the two antennas in addition to the plane their vibration factor outside, other factors caused by the motion state is consistent.

The airplane actual flight state of stress diagram will be given at first:

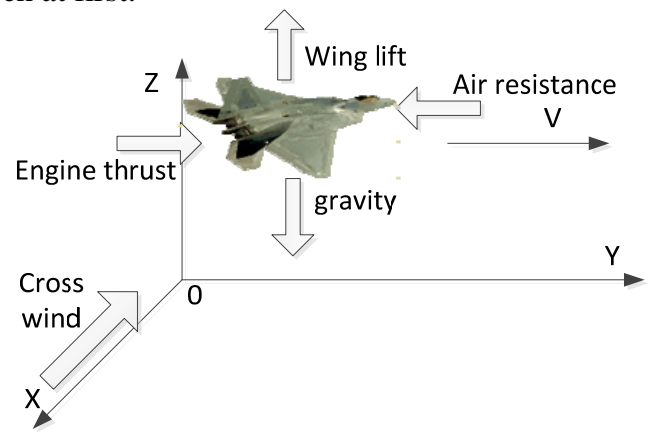

Figure 2. The plane actual flight stressed diagram

The ideal SAR flying conditions, should be in a fixed height $\mathrm{H}$, strictly along $\mathrm{Y}$ direction to speed $\mathrm{V}$ flight uniform motion in a straight line, and the actual state is affected by the change of airflow disturbances, air resistance, wing lift and cross wind blows will change the stress of the plane, the plane is difficult to maintain inertia motion, so the $\mathrm{X}, \mathrm{Y}, \mathrm{Z}$ three axis may have a new sport.

We only analysis to the speed for a step change, $\mathrm{Y}$ direction speed change affect is the SAR Doppler modulation frequency, for is side-glance system for Doppler center frequency have no effect on always is 0 , it affects only SAR image focusing effect, because the two antennas placed along the fuselage. Y direction speed change on two channel data influence is consistent, therefore, two channel image motion compensation are consistent; For interferometric phase value for, because the motion state change consistent, movement path to the same, it will not have influence.

$\mathrm{X}$ direction speed change, the influence of the SAR is the equivalent oblique Angle of view, ideal condition $\mathrm{X}$ direction speed is 0 , radar working in is side-glance conditions. Once the $\mathrm{X}$ direction appeared movement speed, namely aircraft fuselage in the $\mathrm{X}$ direction have translation motion component, and the perspective of antenna or vertical plane of the fuselage, this caused the SAR equivalent work in certain Angle of state, this will affect the SAR of the Doppler center frequency and Doppler modulation frequency, affects the image geometric position and focusing effect; And double antenna interference principle is through the delay or displacement makes different equivalent antenna phase center in the space has the same observation point, so that two images on the static goal can be completely consistent because phase and cancellation off, and the premise is the two equivalent phase centers in the space must be a real coincidence point. And because cross wind action led to the body in the X direction translation, after a antenna phase center in track direction before the arrival of a antenna phase center position, due to the $\mathrm{X}$ direction translation makes its in vertical path up the previous one antenna phase center has a mobile quantity, that is to say the space two antenna phase center can never coincidence. And in the process of ATI, whether time domain signal delay interference or complex image registration interference, the work is position transformation operation, itself does not change the echo signal due to space geometrical relationship decision phase, so this part of the interference phase value must be through the other way to compensate.

$\mathrm{Z}$ direction speed under the ideal condition also is 0 , the direction of the movement and $\mathrm{X}$ direction in the influence of interference phase value have the same phase value; In imaging, the sports not only changed the Doppler center frequency and Doppler modulation frequency, in fact also caused the imaging of highly distortion, imaging time also need to consider, but this part of the influence of the two channel for imaging is completely consistent.

\section{THE NONIDEAL STATE OF MOTION OF SAR-ATI INFLUENCE SOLUTION}

The previous analysis can see, not ideal for SAR motion state of the influence is mainly embodied in two categories, one is the imaging quality, and the second is interference phase value.

For airborne SAR real data imaging motion compensation method, there has been a large number of scholars at home and abroad were further research work ${ }^{[8-10]}$, in the focus to solve the influence of interference phase value.

By the analysis of the front, after the registration of the interference is actually two of the complex image corresponding scattering point and imaging antenna phase center when the wave equation phase difference. The ideal state is two antenna phase center in space have totally coincidence observation point. Therefore imaging time corresponding point of phase exactly the same causing interference phase is 0 , and the actual situation is due to the 
antenna phase center in space is not really coincidence point, after a antenna phase center in the track up before the arrival of a antenna phase center position, the other two direction happened migration, as below:

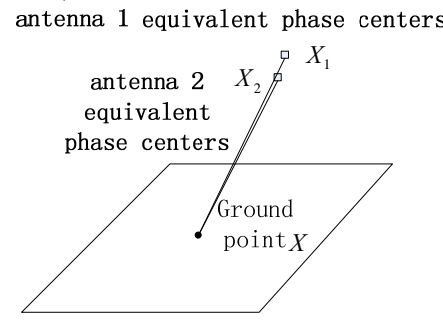

Figure 3. two antenna interference phase as the center and ground point position

As you can see, his ground point $X$ to two antenna equivalent phase centers of norm can be recorded as $\| X_{1}-$ $X \|$ and $\left\|X_{2}-X\right\|$, point $X$ to two antenna phase center norm difference for:

$$
R_{X}=\left\|X_{1}-X\right\|-\left\|X_{2}-X\right\|
$$

It is not difficult to get there next type was established:

$$
\left\|R_{X}\right\| \leq\left\|X_{1}-X-\left(X_{2}-X\right)\right\|=\left\|X_{1}-X_{2}\right\|
$$

And $\left\|X_{1}-X_{2}\right\|$ represent is after image registration, two antenna equivalent phase centers of norm, it is a path to translation cause. If the distance is very small, less than the wavelength $\lambda$ of a small percentage, so the ground all point interference phase error value in a few points. so the ground all point interference phase error value in dozens of $4 \pi$, considering the ground clutter the relative motion of the ${ }^{[11]}$, this part of the influence in the blind speed inside, can not to consider. And usually antenna along the path to baseline length is in the $\mathrm{m}$ magnitude, translation cause speed is usually flight speed of one of the ten part to dozens of, so in vertical direction of displacement is usually a few centimeters. For decimeter wave speaking, phase change to a few points $\pi$, for centimeter-wave speaking, phase change is more big, therefore, must consider the change of phase.

Due to the global norm change is bigger, therefore can only consider local norm and the variation of, a ground point $X$ around a bit $X+\mathrm{d} X$, point to two phase center norm difference for:

$$
R_{X+d X}=\left\|X_{1}-X-d X\right\|-\left\|X_{2}-X-d X\right\|
$$

As you can see, $\triangle R_{\mathrm{X}}=\triangle R_{\mathrm{X}+\mathrm{dX}}-R_{\mathrm{X}}$ and antenna phase center, ground point itself in banach space location, $\mathrm{d} X$ direction vector is different, $\triangle R_{\mathrm{X}}$ change is different, can get proof, $\mathrm{d} X$ keep the same direction vector, $\|\mathrm{d} X\|$ increase, $\left\|\triangle R_{\mathrm{X}}\right\|$ is drab and not reduced.

Therefore, we can consider to complex interference image area limits, in each area choose a point of reference $X$, the other point relative to the reference point $\left\|\triangle R_{\mathrm{X}}\right\|$ restrictions in the wavelength of a certain threshold of less than, to ensure that each group interference image all static target point interference phase variation in blind speed limit corresponding phase within the interval.
This method solved caused by $\triangle R_{\mathrm{X}}$ a static target interference phase change, and will be caused by $R_{\mathrm{X}}$ the target of interference phase have unified increment $\Phi$, this is equivalent to the ideal of the SAR-ATI interference phase profile conducted an $\Phi$ Angle rotation, phase center of mass by the ideal state of the 0 to, because in its IMU is difficult to real-time accurate given $\mathrm{X}, \mathrm{Z}$ direction of motion state, so the direction of displacement can not be directly get, can only use estimation method to solve phase center of mass.

According to the analysis of the literature [8], the ideal of the SAR - ATI Interference phase diagram distribution should be determined by the noise, noise, dynamic goal 3 part of the interference phase composition. Noise interference phase distribution $\operatorname{in}[0,2 \pi$ ), in the ATI detection set when the amplitude threshold will filter out, here, is also the first through the receiver letter mixed ratio and requirements of the false alarm probability setting threshold will its filtering; Secondly, calculating the residual all point of interference phase value, the average for judgment of the distribution of the phase center will be its preliminary as phase center of mass, then the phase center of mass as the foundation, setting moving target detection phase threshold, threshold choice to system minimum radial detectable rate of standard, filtering beyond the threshold of all point to the rest of the point (think is still targets); the average phase again, this time get can think is interference phase center of mass. This method the default a premise is the ground the existence of a large number of is still targets, the proportion of small moving target.

Get in the phase center of mass, will be interference phase diagram of interference phase center of mass compensation to 0 , the moving target interference phase is a perfect target motion caused by the.

\section{ENGINEERING PROBLEMS TO THE INFLUENCE OF THE SAR -ATI}

The airborne SAR-ATI in practical application and the problems mainly has two, one is antenna assembly problem, the other is a two channel sampling clock incomplete synchronization issues.

Theoretically double antenna installation must strictly according to the fuselage direction installation, in fact, due to the error of the assembly, two antenna phase center in the often along the track (body) the direction existing position is poor, the SAR-ATI effect and previously mentioned not on the course of the movement of the agreement, the solution is the same.

In order to ensure two receiving channel signal coherent, two channel sampling clock all will come from the same frequency heald, sampling initial pulse is from the same signal, because the two AD interface card position is not the same, antenna phase center is not same, signal transmission line length is generally not the same, Sampling receiving control signal, antenna receiving signal in the cable is the speed of light transmission, Ground scattering body echo signal is transmitted at the speed of light, cable on degree increased signal transmission delay, plus two channel AD 
sampling chip possible inconsistency, signal in the sampling time may never be the same, this part in the SAR interference image influence not only the same goal in the distance up position, also can affect SAR-ATI interference phase value. So this part of the effect in the treatment of the process not only should through the registration for solve the distance to the position, also need in the interferometric phase center of mass compensation for interferometric phase value.

\section{CONCLUSION}

This paper focuses on the airborne SAR double channel data using ATI method into the Moving Target Indication process various factors affecting the discussion. distance up on time with offset is mainly composed of engineering application of reason is caused, this part for interferometric phase does not produce influence.

\section{REFERENCES}

[1] Stockburger E.F., Held D.N. Interferometric Moving Ground Target Imaging[C]. The Record of the 1995 IEEE International Radar Conference. USA: IEEE Press, 1995: 438-443

[2] Nohara T.J., Weber P., Premji A., et al. Airborne ground moving target indication using non-side-looking antennas[C]. Proceedings of the 1998 IEEE Radar Conference. USA, 1998: 269-274

[3] Breit H., Eineder M., et al. Traffic monitoring using SRTM alongtrack interferometry[C]. 2003 IEEE International Geoscience and Remote Sensing Symposium Proceedings. France, 2003,(2):11871189

[4] Carnade R.E. Dual Baseline and Frequency Along-Track Interferometry[C]. 1992 IEEE International Geoscience and Remote Sensing Symposium Proceedings. USA, 1992,(2): 1585-1588

[5] Bamler R., Hartl P. Synthetic Aperture Radar Interferometry[J]. Inverse Problemes, 1998, 14(4): R1-R54 